\title{
Driver's various information process and multi-ruled decision-making mechanism: a fundamental of intelligent driving shaping model
}

\author{
Wuhong Wang*, Yan Mao, Jing Jin, Xiao Wang, Hongwei Guo \\ Department of Transportation Engineering, Beijing Institute of Technology, \\ Beijing 100081, PR China \\ Xuemei Ren \\ Department of Automatic control, Beijing Institute of Technology, \\ Beijing 100081, PR China \\ Katsushi Ikeuchi \\ Institute of Industrial Science, The University of Tokyo, \\ 4-6-1 Komaba, Tokyo 153-8505, Japan \\ Received: 25-01-2011 \\ Accepted: 20-04-2011
}

\begin{abstract}
The most difficult but important problem in advance driver assistance system development is how to measure and model the behavioral response of drivers with focusing on the cognition process. This paper describes driver's deceleration and acceleration behavior based on driving situation awareness in the car-following process, and then presents several driving models for analysis of driver's safety approaching behavior in traffic operation. The emphasis of our work is placed on the research of driver's various information process and multi-ruled decisionmaking mechanism by considering the complicated control process of driving; the results will be able to provide a theoretical basis for intelligent driving shaping model.
\end{abstract}

Keywords: Driving behavior, Situation awareness, Intelligent driving assistance, Driver cognition

\section{Introduction}

In real traffic operation, the stimulus to the response is the risk of collision perceived by the drivers based on their driving experiences. Since driver behavior has been playing an important role in traffic operations ${ }^{1}$, it is necessary to analyze driver behavior before modeling of intelligent driving shaping behaviour. As drivers encounter the car-following situation regularly, it was used to research driver's various information process and multi-ruled decision-making ${ }^{2-4}$. In the deceleration and acceleration situation, driver's car-following behavior can be described as the relationship between reaction time and deceleration and acceleration. The old models of car-following behavior have been slightly far away from the real traffic because of the assumption of driver's optimal performance. At present, with the fuzzy logic theory, the analysis of car-following behavior becomes mature ${ }^{5,6}$. Therefore, car-following models are not only the main content in micro traffic simulation models but also the important basis of developing

\footnotetext{
* Corresponding author: wangwuhong@bit.edu.cn
} 
advanced in-vehicle information support systems, which is of great importance to traffic safety and road capacity. However, it's still difficult to exactly describe the driving cognitive in car-following model because the driving actions are of variable in everyday.

Because of the high adaptability and complexity of driver's various information processing, driving situation awareness was presented in car-following process. The definition of driving situation awareness is the perception of the elements in the road environment within a volume of time and space, the comprehension of their meaning, and the projection of their status in the near future. It involves navigation knowledge, environment and interaction knowledge, spatial orientation knowledge and vehicle status knowledge in driver situation awareness ${ }^{7}$. As the driving situation awareness is complex, there is no normative model of driving behavior. But in special circumstances, it is more effective to set a driving behavior model in carfollowing. Therefore, in order to improve the carfollowing behavior model, it is important to incorporate variable intentional and unintentional sources in it. Because of the various information processing, drivers are under a lot pressure, as such it is necessary to develop driver assistance system to help drivers. Adaptive Cruise Control (ACC) is one of the highlighted example of driver assistance systems ${ }^{8,9}$.

In various information processing stages such as perception, decision making and execution ${ }^{2,20}$, drivers are easily to make mistakes because of the frequent operations about acceleration and deceleration which may cause accidents. However, ACC can reduce not only the rear-end collision but also driver's workload because of its automation of the longitudinal vehicle control. Therefore, ACC is designed for flowing traffic flow and adjusts the speed according to traffic conditions. On the other hand, it is difficult to describe the driver's multi-typed information process and multiruled decision-making mechanism by using analytical methods. Based on the fuzzy logic theory, drivers depend on the multi-typed information integrated with different weights. There will be multi-inputs and single output in driver's car-following behavior, as a result, it is difficult to describe driver's deceleration and acceleration behavior.

The main objective of this paper is to discuss driver's various information process and multi-ruled decision- making mechanism in car following process, which is a fundamental of building intelligent driving model. This paper is structured as follows: the next section reviews driving behavior analysis in traffic operations. It is followed by the discussion of driver's acceleration and deceleration behavior in car following mode. Then it describes driving human factors which affects the acceleration and deceleration situation in section 4 . In section 5, driving situation awareness is introduced in car-following process. In section 6, the application of ACC is discussed and the development of ACC is briefly mentioned. After that we describe various carfollowing models, and then present driver's various information process and multi-ruled decision-making mechanism. Finally, some concluding remarks and future works are summarized.

\section{Driver behavior analysis in traffic operations}

Traffic system involves drivers, vehicles, roads and environment and their interactions. Mc Call and Traivei ${ }^{1}$ described the three components as: roads and environment includes infrastructure in the road and the situation of dynamic climate; vehicle is an equipment of moving which includes telematic devices and infotainment gadgetry; driver plays an important role in human-vehicle system which needs to be operated safely. Driver's age, gender, attitude to driving, driving skills and range of visibility are involved in driver factors. The vehicle factors are influenced by the design and types of vehicles, vehicles' performance and maintenance and repairs. Road factors include the capacity, geometry, surface of road and markings, signs and signals in the road. Environment such as weather, light, density and policies also plays an important role in traffic system ${ }^{10}$. However, drivers are the core of road traffic safety in traffic operating ${ }^{11}$, so it is necessary to analyze drivers' behaviors in detail.

When driving a vehicle in roadways, the driver will percept various information by eyes, ears, etc. Then the driver acquires sufficient information concerning the driving task to allow the consideration of an appropriate response. After analysed the information, the driver makes a decision about how to respond for a safety driving. Finally, the driver manoeuvres the vehicle into acceleration, deceleration, braking or swerving ${ }^{10}$. Furthermore, driver's behavior interacts with traffic 
operations. For example, when a driver makes mistakes in information processing, it may lead to accident or congestion in the traffic system. But if the traffic system is in the congestion conditions, the driver will decelerate to a lower speed to adapt to the situation. The relationships among the three components in traffic system are shown in Fig. 1. There are two essential vehicle movements with longitudinal motion and lateral movement, when a car is running on the roadways. Correspondingly, drivers also have two main behaviors, lane-changing and approaching. In our research we just discuss the approaching behavior in car-following situation, which involves deceleration and acceleration situations.

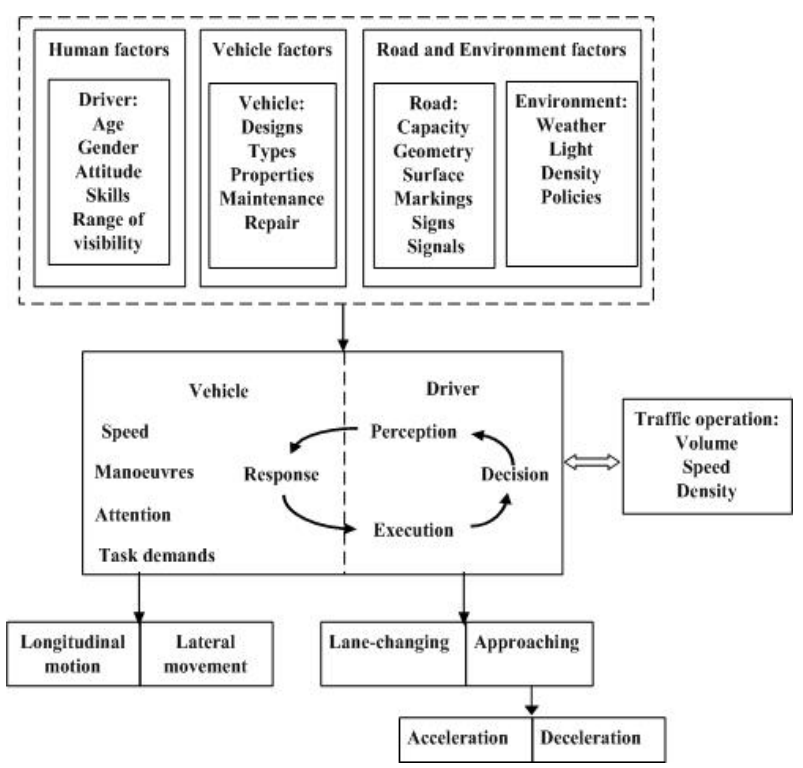

Fig.1. Drivers, vehicles, roads and environments

\section{Driver's deceleration and acceleration behavior}

It is unbelievable to investigate the car-following models without considering driving human factors ${ }^{3,4}$, where a driver controls the brake and accelerator in order to maintain an acceptable distance behind a leading vehicle in the same lane. Driving is considered to be a complex task requiring perception, comprehension and projection of states of the roadway environment, as well as decision making on courses of action and execution of driving behavior.
As shown in Fig. 2, the vehicle of interest, car A, is following leading vehicle, car $\mathrm{B}$, in the right lane of a divided road. Car A would like to follow car B with a safety distance ${ }^{12,13}$. The decision on whether or not to follow depends on a large number of factors including: the distance between cars $\mathrm{A}$ and $\mathrm{B}$, the speeds of cars $\mathrm{A}$ and B, car A's preferred velocity, and the traffic flow characteristics in the area, and so on. Drivers encounter such situations regularly and make good decisions with little conscious thought.

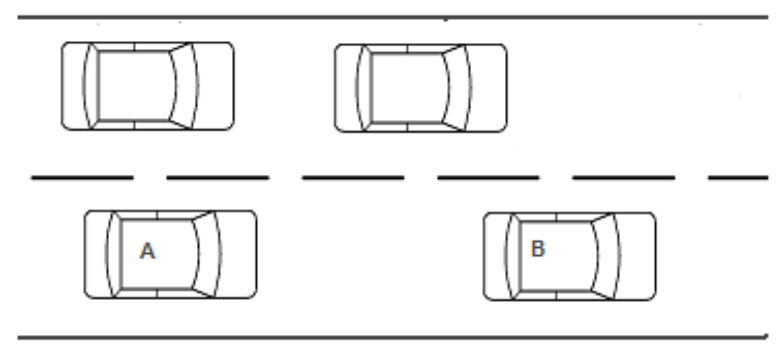

Fig.2. Driver's car-following behavior with various traffic situations

The traffic situation in which the driver evolves plays a crucial role in determining the type of driver's following actions. Driving takes place in a wider context in which the driver constantly interacts with road environment and the vehicle. In actuality, a driver does not directly respond to the relative velocity between the vehicles, but rather to the amount of risk of rear end collision that he perceives from the spacing, the velocity, and the probability of a collision. Accordingly, the stimulus to the response in the car following situation is the risk of

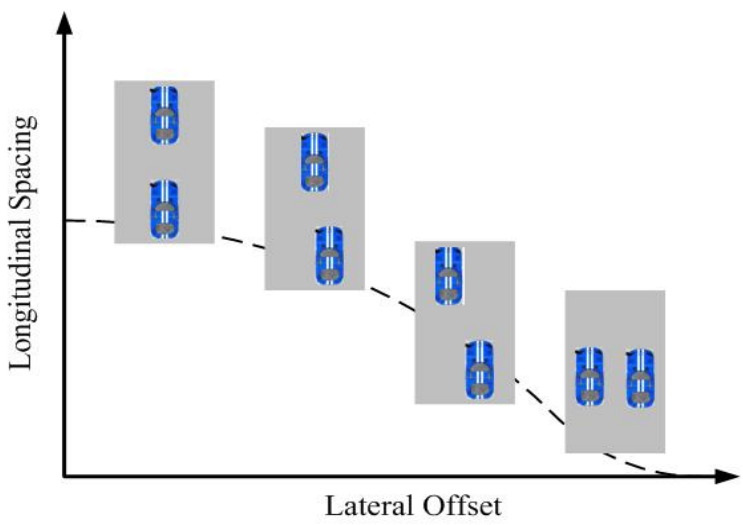

Fig.3. Relationship between horizontal separations of two consecutive vehicles and following distance ${ }^{13}$. 
a rear end collision perceived by the drivers on the basis of their driving experiences. In actual traffic, the following distance between the two consecutive vehicles is a function of the width of the escape route and the amount of off-centeredness between them ${ }^{13}$. Figure 3 demonstrates an approximate shape of the relationship between horizontal separations of two consecutive vehicles and following distance ${ }^{14}$.

\section{Driving human factors of influencing driver's deceleration and acceleration behavior}

The drivers are subjected to many different traffic situations; the most frequent situation is the one where the drivers are forced to follow another vehicle in urban city. The car-following situation in urban traffic is characterized by the headway (time or distance between two consecutive vehicles) and the degree to which the following vehicle tracks the velocity changes of the leading vehicle. During this process, if the driver recognizes high risk, the driver would decelerate quickly; if the driver recognizes low risk, and then decelerate slowly. If the leading car decelerates, the following car driver would select a proper reaction time and deceleration rate to prevent rear end collision according to his/her driving experiences. Hence, all drivers have their own reaction area with respect to reaction time and deceleration rate, and they follow the leading vehicle to a proper distance considering their reaction area. Drivers in car-following process are not likely to resort to sudden accelerations or decelerations except in emergencies. Accordingly, the driver's carfollowing behavior in deceleration and acceleration situation can be expressed by the relationship between reaction time and deceleration and acceleration.

Driving behavior is completely rational, drivers are perfectly able to perceive distance, speed and acceleration, so the drivers not only process the traffic information but also adjust and control the vehicle, and the effect of driving behavior on the traffic flow becomes the core of road traffic safety. Since car following models are able to describe the longitudinal speed-distance relationship between leading vehicle and following vehicle in the same lane, it can determine the acceleration (or deceleration) rate of the following vehicle in a given time interval based on the actions of the leading vehicle(s). Once the acceleration or deceleration rate of the following vehicle is determined, the motion equations are used to compute the speed and the position of the following vehicle for any given time interval. Although the models can describe the carfollowing behavior to a certain extent, the deficiency still exits, in particular, it is difficult to describe the driving cognitive behavior in car-following objectively and correctly. As a matter of fact, if drivers are limited to a single lane, there will be a vehicle in front of them and the physical properties of each are known, so the form of descriptive equation should capture the driver behavior observed.

Drivers could drive well enough to accomplish their tasks in most cases, but sometimes they do not seek continual improvement in driving skill towards some normal optimal levels. Therefore, those assumptions for modeling driver car-following behavior have gone too far from the real cases, the main problem with a number of existing models is that they frame the question of driving behavior around the idea of optimal performance. However, fuzzy logic allows the introduction of a quantifiable degree of uncertainty into the modeled driving car-following process in order to reflect natural perception of real variables in traffic operation. This is accomplished by dividing the parameter space of real world variables, e.g. speed, headway, into a number of overlapping sets and associating each one with a particular term, e.g. "close", "very close" 5,6. At present car following models form one of the main processes in all microscopic traffic simulation modeling and ITS development, and some new progresses have been developed in driving behavior analysis and modeling for application into traffic safety and traffic flow.

It is extremely difficult to exactly describe driver's carfollowing behavior from either engineering or psychological viewpoint because of the high adaptability and complexity of driver's perception, decision-making and execution stage $\mathrm{e}^{20}$. Every specific factor that may affect the driver's car-following behavior has been deeply studied, among which the headway is regarded as the main weigh indices, the headway increases with driver's age and generally males adopted shorter headways than females ${ }^{4}$. Evans also found that the headways are short for drivers who do not wearing seat belts ${ }^{4}$. It is found that, in heavily congested conditions in which drivers are forced to 
respond closely to lead vehicles for extended periods, drivers may adopt uncomfortably short following distances to prevent vehicles in adjacent lanes from entering the gap immediately in front of their vehicle. As a result, driver's car-following behavior may be characterized by an increasing number of attention lapses, resulting in vehicle control errors (e.g. dangerously short headways) ${ }^{3}$. Consequently, development of car-following model should also consider from understanding mechanisms of driver's controlling vehicle errors.

\section{Driving situation awareness in car-following process}

The competence to a task-specific understanding of the working situation is termed as situation awareness ${ }^{15}$. Situation awareness was first presented in connection with pilot performance in air-to-air combat and the ability of commercial airline pilots to fly in difficult air traffic condition ${ }^{16,17}$. In general, driving like flying can be thought as a dynamic control system in which the input variables can change over task time. The input variables such as roadway conditions, weather conditions, vehicle conditions, and driver conditions are primarily traffic events and/or incidents with some degree of uncertainty. Based on information detected on the state of the traffic environment, drivers select courses of action that may or may not change traffic operation. Driver actions can include slowing down, accelerating, passing a vehicle, turning, etc. Under such circumstance, subsequent studies have also applied the principles behind situation awareness into driving in traffic $^{7,12,13,18}$. Ma and Kaber ${ }^{17}$ summarized underlying factors in driver situation awareness, including navigation knowledge, environment and interaction knowledge, spatial orientation knowledge and vehicle status knowledge. They suggested an integration of these forms of knowledge in a model of driver information processing to achieve accurate situation awareness in traffic operation (see Fig. 4).

Using the principal ideas underlying the models of cognition adopted by Endsley ${ }^{14}$ and considering the variations in traffic operations, driving situation awareness could be defined as the perception of the elements in the road environment within a volume of time and space (level $1 \mathrm{SA}$ ), the comprehension of their meaning (level 2 SA), and the projection of their status in the near future (level $3 \mathrm{SA}$ ).

From the viewpoint of the driver's car-following behavior with situation awareness, drivers are the most complicated core part of the traffic system, they have to perform the task of information processing, decisionmaking, vehicle adjusting and control almost at the same time. They firstly obtain traffic information from road environment and vehicle operating conditions, then feed all the correlative rules to their brain and make decisions instantly. However, it is the most requiring job for drives to be ready at any minute for the latent danger that might suddenly show up in the driver's visual field and will almost attract all attention at once. As a result, some advanced assistant systems have been designed to support drivers in maintaining some safety thresholds or ensuring compliance with some formal driving rules (e.g. maintaining safe time headways in car-following situations) ${ }^{19}$.

\section{Analysis of Adaptive Cruise Control(ACC) strategies}

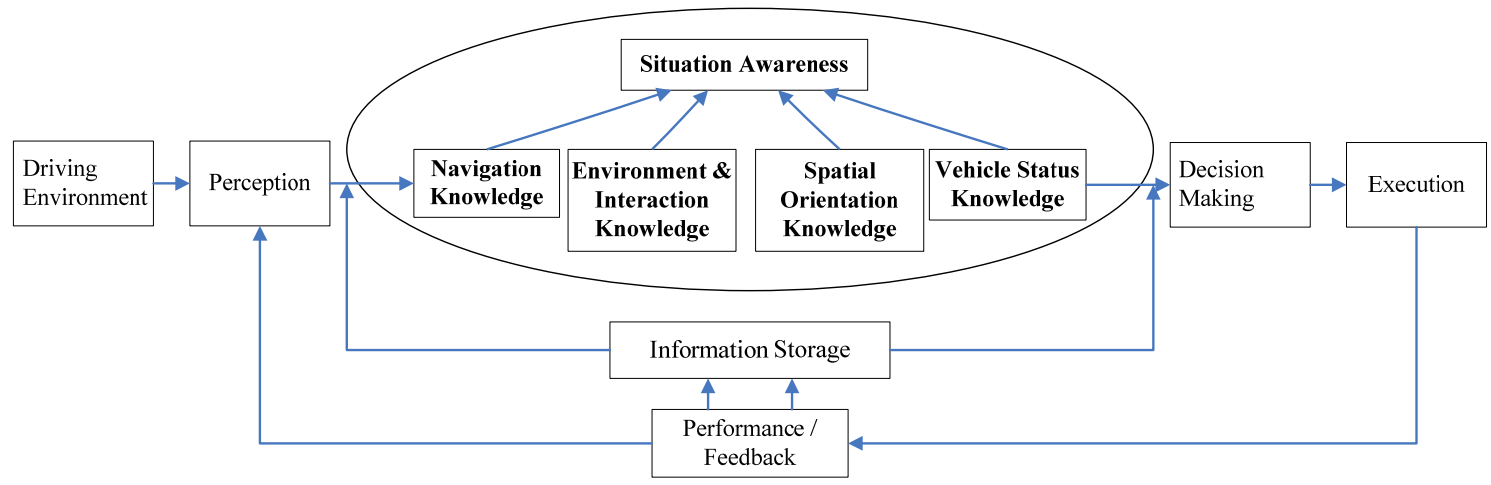

Fig.4. Driver situation awareness in traffic operation ${ }^{17}$ 
Driving behavior has been becoming increasingly complex in urban traffic; it is very difficult to realistically measure the driver's approaching safety behavior because some advanced vehicles have been equipped with an ACC system ${ }^{8,9}$. The headway is kept by adjusting the speed of the car to prevent exceeding a programmed headway of the system dependent on speed. In fact, the ACC can accelerate and/or decelerates the vehicle automatically to keep the target velocity and the headway distance set by driver (Fig. 5). The ACC is a system that does not communicate with other vehicles or roadside systems. Therefore the ACC is applicable in mixed traffic flows: vehicles with and without the system can use the same road.

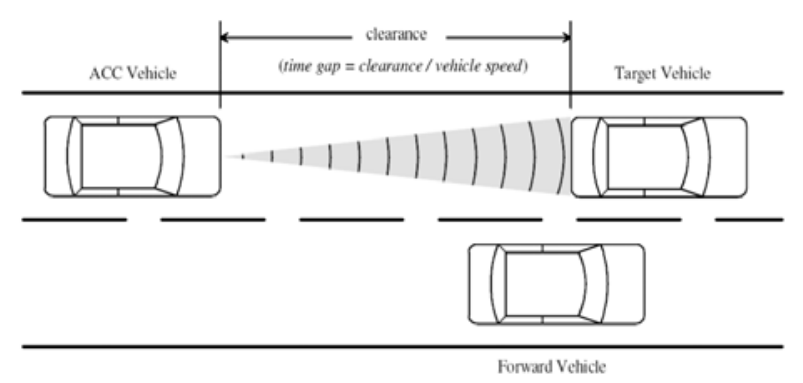

Fig.5. Operation of Adaptive Cruise Control system in traffic system

Driver operates the ACC via a set of switches on the steering wheel. If a slower moving vehicle is detected, the ACC system will calculate its location, movement and relative speed, and slow the vehicle down and control the clearance, or time gap, between the ACC vehicle and the forward vehicle. If the system detects that the forward vehicle is no longer in the ACC vehicle's path, the ACC system will accelerate the vehicle back to its set cruise control speed. This operation allows the ACC based vehicle to autonomously slow down and speed up with traffic without intervention from the driver. However, when drivers drive vehicles to run on road, drivers set the ACC at higher speeds and at shorter headways compared to unsupported driving. Drivers have poor control over lane position with ACC compared to driving without. It was concluded that the ACC was used in a manner that may improve traffic flow and harmonization, but only if higher speeds and shorter headways do not increase the rate or severity of accidents. In the absence of a leading vehicle the ACC controls speed by keeping the actual speed of the vehicle equal to the reference speed as set by the driver. If a lead vehicle is detected the ACC automatically switches to headway control, which means keeping the actual preset default time headways.

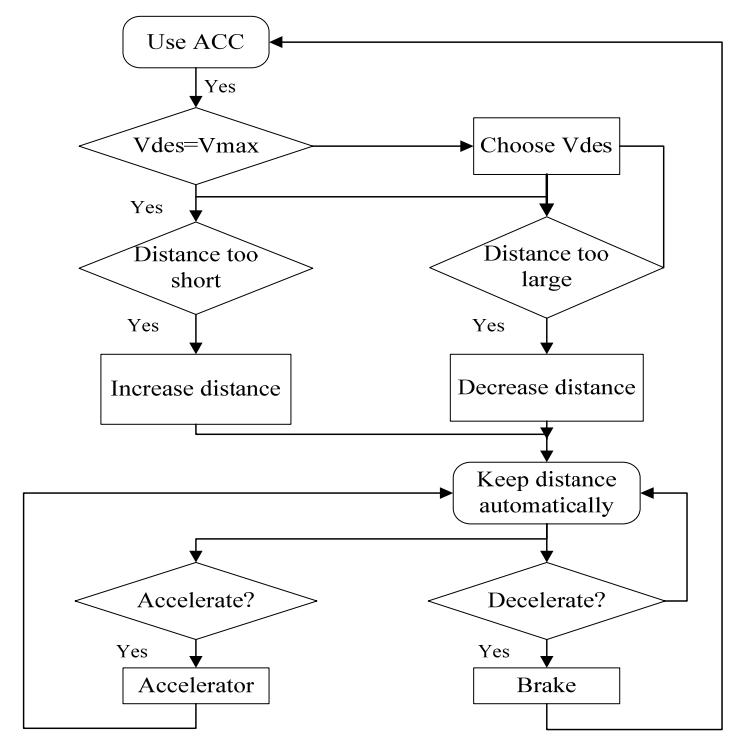

Fig.6. Technical functions of adaptive cruise control

The method by which the ACC based vehicle's speed is controlled is via engine throttle control and limited brake operation. During operation of ACC system, it will send a target speed to the engine control module and deceleration commands to the brake control module to maintain the set time gap between the vehicles. The ACC system is expected to reduce not only rear-end collision accidents caused by the driver's error in the processes of perception, decision making and execution stage, but also driving workload because of the reduction of the frequent operations about acceleration and deceleration. If a vehicle is detected in the lane ahead, the ACC system adjusts the speed of vehicle by slowing the engine or by gently applying the brakes, and then holds the appropriate constant distance. The driver can choose between three different settings for constant distance. If the vehicle ahead happens to brake suddenly, the driver has to apply the brakes. Because the radar beams are cone-shaped, and thus not very wide directly ahead of the car, the driver needs to be aware of other vehicles that pull out suddenly in front. Accordingly, the ACC system automates the longitudinal vehicle control 
partially to reduce driver's workload.

Since ACC is designed to adjust the vehicle's speed according to traffic conditions, it means that nonmoving objects, such as vehicles in a traffic jam, are not picked up by the system. Therefore, the control rules of ACC system that are already implemented by many automobile manufacturers differ from each other. Especially the ACC system should be considered while study the car-following safety behavior in congested traffic, as a result, it is much necessary to develop carfollowing safety algorithm based on adaptive cruise control. Fig. 6 shows the technical function of ACC during the process of controlling vehicle, its system states are divided into ACC off state, stand by state and active state. Though ACC has an additional function of car following when the preceding vehicle is driven at a speed lower, one new importance enhancement is currently the car following capability at low speeds including stop-and-go capability.

\section{Driver's various information process and multi-ruled decision-making mechanism}

Because of the complexity of driving situation awareness, everyday driving involves a seemingly endless variety, it is not practical to set up a normative model of driving behavior ${ }^{20,21}$. Instead it is more effective to model driving behavior in special circumstances with basic model describing driver's carfollowing behavior, where a driver controls the brake and accelerator in order to maintain an acceptable distance behind a lead vehicle in the same lane. When modeling driver's car-following behavior, it must be improved by incorporating these intentional and unintentional sources of variability.

From the cybernetic viewpoint, a driver is an intelligent agent with a number of fuzzy regulations and adaptability, which lead to develop very complicated logicality and new strategies for new situations in driving $^{22}$. Different from classical control process, there is quite multi-inputs and single output in driver's car following behavior. Drivers don't depend on the final execution on any of the inputs, but on the multi-typed information integrated with different weights. These weights are specified according to drivers and fluctuate with driver's attention that is influenced by the driving purpose, roadway shape, relative velocity, traffic volume, willingness to follow and driving time. Besides, these weights also fluctuate with the physical condition in car following and increase sharply for emergencies. That is, a driver is subconsciously sensitive to some emergencies, whose appearance will attract the driver's attention immediately after pre-treatment, and it will be given high priority and processed firstly due to its great urgency. This is the reason why the driver is very adaptive to emergent traffic situation.

Driving is an extremely complicated control process, where driver performs various driving tasks for keeping vehicle safe based on all kinds of information which is captured mainly by eyes. Since the ever-increasing variety of driving situations, including changes in the driving task associated with the increasing number of different in-vehicle information support systems, the corresponding variety of skills and abilities of driver is required. During driver's car-following process, for instance, drivers take the relative velocity and the following distance as inputs for the central processing unit that makes predictions and gives out guidance for safety. However, in the real traffic conditions drivers make use of over two inputs. In the case of a vehicle approaching or departing from another vehicle, much traffic information can be perceived by drivers, including changes in the following distance, those on the visual angle subtended by the vehicle ahead, changes of the front vehicle's position on the back vehicle's windscreen, those on the visual angle subtended by the road, and those of the relative position between two cars.

Drivers will choose some kind of information from urban traffic as input in car following depending not only on driver's cognitive status and attention distribution, but also more on the car following conditions (the headway and relative velocity). As the leading vehicle is still far from it, the driver may identify the distance ahead by the leading vehicle and make predictions according to the changes in the apparent size. While the leading vehicle is near to it, the driver may judge by the visual angle subtended by the road ahead. At high speed, drivers may concentrate much more to observe the leading vehicle's taillights through red flash warn the back driver to be ready to decelerate. While at low speed, driver may allocate more attention to capture changes in the visual angle subtended by the leading vehicle. Visual search in 
driving involves identifying highlighted traffic information in a constantly changing, moving scene. For example, regulatory signs occur in fairly predictable locations and contain some traffic information that is highly predictable. In contrast, changeable message signs may be located at less predictable location and may contain less predictable information (e. g. temporary speed limit, lane closed).

\section{Car-following models based on driver behavior}

Many car-following models, which all discussed the longitudinal movement of two successive vehicles in the microscopic traffic stream, have been developed based on different assumptions, such as the speed difference with the leader, the difference between the desired headways to the leader. Among these, maybe the GHR model dated from 1950s is nicely famous models, which formulated the relationship between the following vehicle's acceleration and the factors including the follower's speed, the relative spacing and speed of the successive vehicles. Then, other car-following models such as safety distance model, collision avoidance model, linear model, were established. But rarely there are models concerned about the driver factor which varies widely until the construction of psychophysical or action point model (AP). The action point model presented the concept that drivers would initially be able to tell they were approaching a leading vehicle, primarily due to changes in the apparent size of the vehicle, by perceiving relative velocity through changes on the visual angel subtended by the vehicle ahead. As a matter of fact, the action point model needs to further evolve due to its complication.

In order to understand much more about driving shaping behavior related car-following process, many works have done for calibrating the existing models or establishing new models ${ }^{18}$. A more detailed model based on safety approaching behavior was developed with the parameterization of the equations in deceleration and acceleration algorithms by means of trying and testing with simulation as well as the empirical data and the numerical simulation of different driving situations for exploring the variation of spacing and speed with various driving time ${ }^{21}$. Furthermore, some published traffic data from instrumented vehicles were used to test the validity of the visual angle model assumptions; especially the ability of such models in representing the effect of heavy goods vehicles. By comparing the simulated following distances (clear spacing) with those from the data, visual angle models are found to be capable of replicating real traffic movements when both leading and following vehicles are "small cars" 23 .

By using different car-following models to represent different driving styles, the heterogeneity in driver's car-following behavior was discussed, and the research results show that the considerable differences existed between the car-following behaviors of passenger car drivers and truck drivers who appeared to drive in general with a more constant speed and the desired time headways of passenger car drivers were smaller when following a truck than when following another passenger car $^{24}$.

\section{Concluding remarks and future works}

In deceleration and acceleration situation, the relationship between driver's reaction time and approaching behaviour is the main element of driver's safety approaching behavior in traffic operation. Although various car following models have been developed for traffic safety and traffic flow, it is difficult to describe the driving cognitive behavior exactly. However, using modeling of driver's carfollowing behavior and analysis of driving situation awareness, all components of driver's safety approaching model will hopefully not only contribute to develop realistic driver assistance system but also reveal the essence of traffic flow characteristics at the microscopic level.

The definition of driver's various information process and multi-ruled decision-making mechanism is proposed through the human factors analysis of driving behavior in car-following process. However, it should be thoroughly evaluated in a situation where appropriate car-following data can be acquired from field tests with an instrumented vehicle, driving simulator or video observation in the future research. As such the driver's various information process and multi-ruled decisionmaking mechanism should be analyzed further quantitatively; the results will anticipate becoming a fundamental for modeling of driving shaping behavior as to support intelligent driving and improve active safety of the vehicle.

\section{Acknowledgments}

This research was supported in part by National Nature Science Foundation of China under Grant 50878023. 


\section{References}

1. Joel C. Mc Call, and Mohan M. Traivei, Driver behavior and situation aware brake assistance for intelligent vehicles, proceeding of the IEEE, 95(2)2007(2).

2. W. H. Wang, H. Bubb, K. Ikeuchi, Q. Cao, Measurement of dangerous traffic conditions through driving dependability analysis, Journal of Scientific and Industrial Research, 69 (2010) 431-437.

3. T. A. Ranney, Psychological factors that influence car following and car-following model development, Transportation Research Part F, 2 (1999) 213-219.

4. L. Evans, P. Wasielewski, Risky driving related to driver and vehicle characteristics, Accident analysis and prevention, 15 (1983) 121-136.

5. K. Tanaka, An introduction to fuzzy logic for practical applications (Springer-Verlag, New York, 1996).

6. J. Wu, M. Brackstone, M. McDonald, Fuzzy sets and systems for a motorway microscopic simulation model, Fuzzy Sets and Systems, 116 (2000) 65-76.

7. M. L. Matthews, D. J. Bryant, R. D. Webb, J. L. Harbluk, Model for situation awareness and driving, Transportation Research Record, 1779 (2001) 26-32.

8. M. Hoedemaeker, K.A. Brookhuis, Behavioral adaptation to driving with an adaptive cruise control (ACC), Transportation Research Part F 1 (1998) 95-106.

9. B. Arem, C. Driel, R, Visser, The impact of cooperative adaptive cruise control on traffic-flow characteristics, IEEE Transactions on Intelligent Transportation System, 7(2006),429-436

10. W. H. Wang, Traffic Engineering (China Communications Press, Beijing, 2010).

11. W. H. Wang, Wei Zhang, Dehui Li, Kiyotaka Hirahara, Katsushi Ikeuchi, Improved action point model in traffic flow based on driver's cognitive mechanism, Intelligent Vehicles Symposium (2004)447-452.

12. R. Sukthankar, J. Hancock, C. Thorpe, Tactical-level Simulation for Intelligent Transportation Systems, Mathematical and Computer Modeling, 27(9-11) (1998) 19-24.

13. B. Gunay, Car following theory with lateral discomfort, Transportation Research Part B, 41 (2007) 722-735.

14. M. R. Endsley, Situation awareness in dynamic systems, in: Proceedings of the 11th congress of the international ergonomics association, Vol. 1 (Taylor and Francis, London, 1992), pp. 801-803.

15. N. Sarter, D. Woods, Situation awareness-a critical but ill-defined phenomenon, International Journal of Aviation Psychology, 1(1) (1991).

16. R. Small, C. Howard, A real-time approach to information management in a pilot's associate, In: Proceedings of IEEE/AIAA 10th digital avionics system conference (1999).
17. R. Q. Ma, D. B. Kaber, Situation awareness and workload in driving while using adaptive cruise control and a cell phone, International Journal of Industrial Ergonomics, 35 (2005) 939-953.

18. M. Brackstone, M. McDonald, Car-following: a historical review, Transportation Research Part F, 2 (1999) 181196.

19. W. H. Wang, F. G. Hou, H. C. Tan, H. Bubb, A Framework for Function Allocations in Intelligent Driver Interface Design for Comfort and Safety, International Journal of Computational Intelligence Systems, 5(2010), 531-541.

20. W. H. Wang, Q. Cao, K. Ikeuchi, H. Bubb, Reliability and safety analysis methodology for identification of drivers' erroneous actions, International Journal of Automotive Technology, 2010 11(6),873-881

21. W. H. Wang, W. Zhang, H. Guo, H. Bubb, K. Ikeuchi, A safety-based behavioral approaching model with various driving characteristics, Transportation Research Part C, doi:10.1016/j.trc.2011.02.002

22. W. H. Wang, H. W. Guo, H. Bubb, K. Ikeuchi, Numerical simulation and analysis procedure for modelbased digital driving dependability in intelligent transport system, KSCE Journal of Civil Engineering,(2011)15(5),779-787.

23. Saad Yousif, Jalal Al-Obaedi, Close following behavior: Testing visual angle car following models using various sets of data, Transportation Research Part F, 14 (2011) 96-110.

24. Saskia Ossen, Serge P. Hoogendoorn, Heterogeneity in car-following behavior: Theory and empirics, Transportation Research Part C, 19 (2011) 182-195. 